\title{
Preparation of alginate beads containing a prodrug of diethylenetriaminepentaacetic acid
}

\author{
Yu-Tsai Yanga ${ }^{\mathrm{a}}$, Anthony J. Di Pasqua ${ }^{\mathrm{a}}$, Weiling He ${ }^{\mathrm{a}}$, Tsuimin Tsai $^{\mathrm{b}}$, Katsuhiko Sueda ${ }^{\mathrm{a}}$, \\ Yong Zhang ${ }^{\text {, }}$, and Michael Jay ${ }^{\mathrm{a},{ }^{*}}$ \\ aDivision of Molecular Pharmaceutics, Eshelman School of Pharmacy, The University of North \\ Carolina at Chapel Hill, Chapel Hill, NC 27599-7362, USA \\ ${ }^{b}$ Graduate Institute of Biomedical Materials and Tissue Engineering, College of Oral Medicine, \\ Taipei Medical University, Taipei 11031, Taiwan
}

\begin{abstract}
A penta-ethyl ester prodrug of the radionuclide decorporation agent diethylenetriaminepentaacetic acid (DTPA), which exists as an oily liquid, was encapsulated in alginate beads by the ionotropic gelation method. An optimal formulation was found by varying initial concentrations of DTPA pentaethyl ester, alginate polymer, Tween 80 surfactant and calcium chloride. All prepared alginate beads were $\sim 1.6 \mathrm{~mm}$ in diameter, and the optimal formulation had loading and encapsulation efficiencies of $91.0 \pm 1.1$ and $72.6 \pm 2.2 \%$, respectively, and only $3.2 \pm 0.8 \%$ water absorption after storage at room temperature in $\sim 80 \%$ relative humidity. Moreover, Fourier transform infrared spectroscopy showed that DTPA penta-ethyl ester did not react with excipients during formation of the DTPA penta-ethyl ester-containing alginate beads. Release of prodrug from alginate beads was via anomalous transport, and its stability enhanced by encapsulation. Collectively, these data suggest that this solid dosage form may be suitable for oral administration after radionuclide contamination.
\end{abstract}

\section{Keywords}

Diethylenetriaminepentaacetic acid (DTPA); Decorporation; Encapsulation; Alginate beads; Oral drug delivery

\section{Introduction}

Diethylenetriaminepentaacetic acid (DTPA) is a chelating agent that can be employed to enhance decorporation of transuranic elements, e.g., americium (Am), plutonium (Pu) and curium $(\mathrm{Cm})$, from the human body after internal contamination (Bair \& Thompson, 1974). DTPA is typically administered via intravenous (i.v.) injection, thus requiring well-trained medical professionals. In the case of a wide-spread contamination emergency, an oral formulation would be advantageous in that it could be distributed easily and to a large number of patients. DTPA is hydrophilic and highly ionizable and, therefore, its oral bioavailability is very low; it is approximately $1 \%$ in humans (Cassatt et al., 2008). In order to improve its oral bioavailability, we esterified the five carboxylic acid functional groups on DTPA. We previously demonstrated that this prodrug effectively decorporates ${ }^{241} \mathrm{Am}$

() 2012 Elsevier Ltd. All rights reserved.

*Corresponding author at: 120 Mason Farm Road, Genetic Medicine Building, Room 1043, Campus Box 7362, UNC Chapel Hill, Chapel Hill, NC 27599-7362, USA. Tel.: +1 919843 3775; fax: +1 919966 0197. mjay@ unc.edu (M. Jay).. 
from contaminated animals (Sueda, Sadgrove, Fitzsimmons, \& Jay, 2012). The lipophilic prodrug, however, is an oily liquid that is prone to hydrolysis and, thus, relatively unstable at high temperatures and relative humidities (RH). Besides making it more accessible to patients, dispersing said liquid into a solid matrix could also reduce its interactions with the environment and, thus, enhance its stability.

The encapsulation of many different oils, including a-tocopherol (Luo, Zhang, Whent, Yu, \& Wang, 2011), wheat germ, lemon and citronella oil (Chana, 2011), have been reported. A safe and inexpensive strategy for microencapsulation is the preparation of alginate beads. Alginate, an anionic polysaccharide polymer composed of mannuronic and guluronic acid residues, extracted from brown algae (Han, Guenier, Salmieri, \& Lacroix, 2008), is extensively used in encapsulation applications, as it is nontoxic, biodegradable and biocompatible. Alginate forms a gel in the presence of the divalent cation calcium; this process is termed ionotropic gelation (Cekic et al., 2007;Ramadas, Paul, Dileep, Anitha, \& Sharma, 2000).

The aim of this study was to encapsulate DTPA penta-ethyl ester in alginate beads by the ionotropic gelation method to enhance prodrug stability. In addition, a solid dosage form of DTPA penta-ethyl ester would be very desirable in terms of ease of packaging, handling and administering. An optimal formulation was determined by varying the initial concentrations of DTPA penta-ethyl ester, alginate, Tween 80 surfactant and calcium chloride $\left(\mathrm{CaCl}_{2}\right)$. The optimal formulation of DTPA penta-ethyl ester-containing alginate beads was characterized and in vitro release and stability of the prodrug investigated.

\section{Materials and methods}

\subsection{Materials}

Alginic acid sodium salt (from brown algae; viscosity $\approx 250 \mathrm{cP}$; guluronic to manuronic ratio approximately $39 \%$ to $61 \%$ ), Tween $80, \mathrm{CaCl}_{2}$ and a-tocopherol were purchased from Sigma-Aldrich (St Louis, MO, USA), absolute ethanol and acetonitrile were purchased from Acros Organics (NJ, USA) and DTPA penta-ethyl ester was prepared as previously described (Sueda et al., 2012).

\subsection{Preparation of DTPA penta-ethyl ester-containing alginate beads}

Solutions of alginate polymer (1-3\%, w/v), Tween 80 surfactant (1-3\%, w/v) and $\mathrm{CaCl}_{2}(1$, 3 and $5 \%$, w/v) were prepared in distilled water. To $1 \mathrm{~mL}$ of alginate solution was added $400-800 \mathrm{mg}$ of DTPA penta-ethyl ester and $0.5 \mathrm{~mL}$ Tween 80 solution as an emulsifier. The mixture was homogenized for $2 \mathrm{~min}$ at $13,500 \mathrm{rpm}$ at room temperature (RT) and the emulsion passed through a syringe with a 26 -gauge, $3 / 8$ in. needle into a $10 \mathrm{~mL} \mathrm{CaCl}_{2}$ solution, under gentle stirring ( $700 \mathrm{rpm})$ at RT for $10 \mathrm{~min}$. The DTPA penta-ethyl ester alginate beads were then washed three times with distilled water. Encapsulation of DTPA penta-ethyl ester was frozen at $-80^{\circ} \mathrm{C}$ for $2 \mathrm{~h}$ and was subjected to freeze-drying (FTS System, Inc. Biopharm Division, NY). Freeze-drying was carried out under conditions with both primary and secondary drying at a pressure of $100 \mathrm{mTorr}$. In the first step, primary drying was carried out at $-35^{\circ} \mathrm{C}$ for $20 \mathrm{~min},-30{ }^{\circ} \mathrm{C}$ for $20 \mathrm{~min},-25^{\circ} \mathrm{C}$ for $20 \mathrm{~min},-20^{\circ} \mathrm{C}$ for $20 \mathrm{~min},-15^{\circ} \mathrm{C}$ for $20 \mathrm{~min},-10^{\circ} \mathrm{C}$ for $200 \mathrm{~min},-5^{\circ} \mathrm{C}$ for $30 \mathrm{~min}, 0^{\circ} \mathrm{C}$ for $30 \mathrm{~min}, 10$ ${ }^{\circ} \mathrm{C}$ for $300 \mathrm{~min}, 20^{\circ} \mathrm{C}$ for $300 \mathrm{~min}$; this was followed by secondary drying at $25^{\circ} \mathrm{C}$ for 30 $\min$. 


\subsection{Characterization of DTPA penta-ethyl ester-containing alginate beads for selection of an optimal formulation}

2.3.1. Drug analysis-The amount of DTPA penta-ethyl ester in various samples was quantified using a high performance liquid chromatography (HPLC) system (Shimadzu Prominence UFLC fitted with a Corona charged aerosol detector (CAD) (Dionex/ESA, USA)). The column was an Alltima ${ }^{\mathrm{TM}} \mathrm{C} 18(250 \mathrm{~mm} \times 2.1 \mathrm{~mm} ; 5 \mu \mathrm{m})$ (Grace Davison Discovery Sciences) and maintained at $40{ }^{\circ} \mathrm{C}$. The flow rate was $0.20 \mathrm{~mL} / \mathrm{min}$; the mobile phase was water with $0.1 \%$ trifluoroacetic acid (A), acetonitrile (B) and isopropanol (C). The mobile phase mixture (A-B-C) followed a linear gradient from 94:4:2 to 10:60:30 over $6.5 \mathrm{~min}$, followed by an isocratic phase of 10:60:30 for 4.5 min (Sueda et al., 2012).

2.3.2. Loading and encapsulation efficiencies-The freeze-dried beads were weighed (15-20 mg) and then DTPA penta-ethyl ester extracted. The beads were placed in 1 $\mathrm{mL}$ of $20 \mathrm{mM}$ ethylenediaminetetraacetic acid (EDTA) in phosphate-buffered saline (PBS), $\mathrm{pH} 7.2$, and shaken for $10 \mathrm{~min}$ at RT. Note that no HPLC peaks associated with the degradation of DTPA penta-ethyl ester after treatment with EDTA were observed. Nine milliliters of acetonitrile was subsequently added and the resulting suspension centrifuged at $9000 \times g$ for $15 \mathrm{~min}$ at $4{ }^{\circ} \mathrm{C}$ to pellet the alginate polymer from the disrupted matrix. One hundred microliters of this supernatant was added to $900 \mu \mathrm{L}$ of acetonitrile before analyzing by the HPLC-CAD method. The pellet was then washed once and the supernatant was analyzed, but the amount of DTPA penta-ethyl ester found in this fraction was insignificant ( $\$ 0.02 \mathrm{mg}$ ). To quantify the amount of DTPA penta-ethyl ester recovered, a standard curve of DTPA penta-ethyl ester was prepared, and the loading and encapsulation efficiencies determined using the following formulae:

$$
\text { Loading efficiency }(\mathrm{LE})(\%)=\frac{L}{L_{0}} \times 100 \%, \quad \text { (1) }
$$

where $L$ is the mass of DTPA penta-ethyl ester extracted from DTPA penta-ethyl ester alginate beads, and $\mathrm{L}_{0}$ is the mass of the DTPA penta-ethyl ester alginate bead, and

$$
\text { Encapsulation efficiency }(\mathrm{EE})(\%)=\frac{E}{E_{0}} \times 100 \% \text {, }
$$

where $E$ is the mass of DTPA penta-ethyl ester extracted and $E_{0}$ is the theoretical mass of DTPA penta-ethyl ester.

2.3.3. Particle size analysis and morphology-Particle size was determined using a micrometer gauge (Sargent, USA) with an accuracy of $0.01 \mathrm{~mm}$. Twenty samples of the freezedried beads from different formulations were selected and their sizes measured. The shape and surface morphology of the alginate beads were determined by Scanning Electron Microscopy (SEM; Super III A model, International Scientific Instruments, Militpas, CA, USA).

2.3.4. Water absorption-DTPA penta-ethyl ester-containing alginate beads were weighed before and after storage at RT in a RH of approximately $80 \%$ (using a saturated solution of sodium chloride) until the mass became constant. Water absorption capacity of the DTPA penta-ethyl ester-containing alginate beads was calculated using the following equation:

$$
\text { Water absorption }(\%)=\frac{(W a-W b)}{W b} \times 100 \%
$$


where $W_{a}$ is mass of DTPA penta-ethyl ester-containing alginate beads after storage and $W_{b}$ is weight before storage.

\subsection{FTIR spectroscopy}

FTIR spectra of neat DTPA penta-ethyl ester, DTPA penta-ethyl ester-containing alginate beads, blank alginate beads and sodium alginate polymer were obtained using a Shimadzu Prestige-21 FTIR spectrometer. Sixty four scans of each were obtained at a resolution of 4 $\mathrm{cm}^{-1}$ from 4000 to $400 \mathrm{~cm}^{-1}$.

\subsection{Dissolution}

Dissolution studies were carried out using a USP dissolution Type II apparatus (Hanson Research Elite 8, Chatsworth, CA) with a paddle rotation speed of $50 \mathrm{rpm}$ in $900 \mathrm{~mL}$ dissolution medium $(0.1 \mathrm{~N} \mathrm{HCl}, \mathrm{pH} \sim 1.2)$ at $37^{\circ} \mathrm{C}$ for $45 \mathrm{~min}$. DTPA penta-ethyl ester alginate beads were weighed accurately (225-230 mg, containing $200 \mathrm{mg}$ DTPA penta-ethyl ester) and placed in hydroxypropylmethyl cellulose (HPMC) capsules (size: 0), and the capsules were added to the dissolution medium in the apparatus using sinkers. At specific time intervals, an aliquot $(0.5 \mathrm{~mL})$ was withdrawn using a cannula and passed through a 0.2 $\mu \mathrm{m}$ PVDF filter. The DTPA pentaethyl ester content was then determined by HPLC-CAD. No residual DTPA penta-ethyl ester was found on the PVDF filter. The dissolution study was performed in triplicate and the calculated mean values of cumulative release of prodrug were used for plotting release curves.

\subsection{Stability studies}

DTPA penta-ethyl ester can undergo oxidation, which results in a change of color. In order to maintain the stability of DTPA pentaethyl ester, $0.6 \%$ of the antioxidant a-tocopherol was included in neat DTPA penta-ethyl ester and in the optimal formulation of DTPA pentaethyl ester-containing alginate beads (formulation D3). After preparation, samples were placed in HPMC capsules (size: 0$)$ and kept in refrigerated $\left(4{ }^{\circ} \mathrm{C}\right)$, RT $\left(25^{\circ} \mathrm{C} / 80 \% \mathrm{RH}\right)$ or accelerated $\left(40{ }^{\circ} \mathrm{C} / 80 \% \mathrm{RH}\right)$ storage for three months. The remaining intact DTPA pentaethyl ester was determined using the HPLC-CAD method.

\subsection{Statistical analysis}

Data are presented as the mean \pm standard deviation (STD). Each experiment was repeated at least three times and an unpaired $t$ test was used to establish the significance of differences among groups. Differences were considered statistically significant if $p<0.05$ $(*)$.

\section{Results and discussion}

\subsection{Appearance and morphology of DTPA penta-ethyl ester-containing alginate beads}

A photograph of blank and DTPA penta-ethyl ester-containing alginate beads after freeze drying are shown in Fig. 1A. The DTPA penta-ethyl ester-containing alginate beads are yellow, due to the encapsulation of DTPA penta-ethyl ester, which is a yellow oily liquid (Fig. 1A, left). The alginate beads were spherical and smooth, but after freeze-drying, both blank and DTPA penta-ethyl ester-containing alginate beads had irregular shapes and surfaces that were not smooth. In the SEM image of a DTPA penta-ethyl ester-containing alginate bead after freeze drying (Fig. 1B), the irregular surface can be easily seen. Mean particle sizes of the DTPA pentaethyl ester containing alginate beads are shown in Table 1 and were in the range of $1.58-1.63 \mathrm{~mm}$. No significant differences in particle size were observed among the various formulations. 


\subsection{Characterization of DTPA penta-ethyl ester-containing alginate beads for selection of an optimal formulation}

Various components of the alginate bead formulation were varied to determine an optimal solid dosage form for subsequent studies. It was previously demonstrated that a single 40 $\mathrm{mg} / \mathrm{kg}$ oral dose of DTPA penta-ethyl ester improved decorporation of ${ }^{241} \mathrm{Am}$ from contaminated rats, compared to a no-treatment control group (Sueda et al., 2012). High loading and encapsulation efficiencies were sought to accommodate a large dose of the prodrug. Low water absorption is favored because ester functional groups on the DTPA prodrug can be hydrolyzed (Waterman et al., 2002). Therefore, the percent water absorption in the formulations was also determined.

Increasing the concentration of $\mathrm{CaCl}_{2}$ from $1 \%$ to $5 \%(\mathrm{w} / \mathrm{v})$ at fixed alginate, Tween 80 and prodrug concentrations resulted in decreased loading and encapsulation efficiencies and increased water absorption (Table 1; A1-3). This is consistent with previous reports that showed that increasing calcium concentration in the formulation can decrease encapsulation of the active compound in alginate beads (Østberg \& Graffner, 1994;Pongjanyakul \& Rongthong, 2010). Furthermore, it is known that $\mathrm{CaCl}_{2}$ readily absorbs moisture from the air.

Increasing the concentration of alginate from $1 \%$ to $3 \%$ (w/v) at fixed $\mathrm{CaCl}_{2}$, Tween 80 and prodrug concentrations resulted in no change in the loading efficiency, but higher encapsulation efficiencies and increased water absorption (Table 1; B1-3). Others have shown that encapsulation of a compound in alginate beads increases with increasing concentrations of alginate (El-Kamel, Al-Gohary, \& Hosny, 2003;Manjanna, Pramod Kumar, \& Shivakumar, 2010). The increased water absorption was probably due to the hydrophilic nature of the alginate polymer.

Tween 80 was used here to emulsify the lipophilic DTPA pentaethyl ester and the alginate solution. Table 1(C1-3) shows the effects of varying the concentration of Tween 80 on loading and encapsulation efficiencies and water absorption. The higher loading and encapsulation efficiencies were observed when using $2 \%$ Tween 80 ; no differences in water absorption were observed. The effects of varying the prodrug concentration on loading and encapsulation efficiencies and water absorption are shown in the Table 1(D1-3). Using higher concentrations of the prodrug resulted in greater loading and encapsulation efficiencies. This is consistent with earlier reports (Hari, Chandy, \& Sharma, 1996;Wan, Heng, \& Chan, 1992) in that increasing the concentration of the active compound in the alginate aqueous phase of the emulsion yielded increased encapsulation. Furthermore, an increase in the concentration of drug in the formulation resulted in a decrease in water absorption.

The D3 formulation was chosen as the optimal formulation and was used in subsequent experiments. Its loading and encapsulation efficiencies were the highest achieved, at $91.0 \pm$ 1.1 and $72.6 \pm 2.2 \%$, respectively. Its water absorption was the second lowest at $3.2 \pm 0.8 \%$.

\subsection{FTIR spectroscopy}

Interactions between drug and carrier can lead to identifiable changes in the FTIR spectrum of a solid dosage form. Thus, FTIR spectra of DTPA penta-ethyl ester, sodium alginate polymer, and blank and DTPA penta-ethyl ester-containing alginate beads were obtained (Fig. 2). The spectrum of DTPA penta-ethyl ester has absorptions at 2983, 1732, 1190 and $1029 \mathrm{~cm}^{-1}$, which correspond to $\mathrm{sp} 3 \mathrm{CH}_{2}$ and $\mathrm{CH}_{3}, \mathrm{C}=\mathrm{O}, \mathrm{C}-\mathrm{O}$ and $\mathrm{C}-\mathrm{N}$ stretching vibrations, respectively. In the sodium alginate spectrum, the absorptions at $3394 \mathrm{~cm}^{-1}$ and $1637 \mathrm{~cm}^{-1}$ correspond to the stretching vibrations of $\mathrm{OH}$ and $\mathrm{C}=\mathrm{O}$, respectively. When alginate beads are formed, the aforementioned $\mathrm{C}=\mathrm{O}$ stretching vibration shifts to a higher 
wavenumber $\left(1738 \mathrm{~cm}^{-1}\right)$ (Sartori, Finch, \& Ralph, 1997). In the FTIR spectrum of the DTPA penta-ethyl ester-containing alginate beads, absorptions associated with DTPA pentaethyl ester are observed that do not differ in position from neat prodrug. Thus, DTPA pentaethyl ester did not appear to react with excipients during formation of DTPA penta-ethyl ester-containing alginate beads.

\subsection{Dissolution}

The in vitro release profile of DTPA penta-ethyl ester in dissolution medium $(0.1 \mathrm{~N} \mathrm{HCl}, \mathrm{pH}$ 1.2) at $37{ }^{\circ} \mathrm{C}$ from DTPA penta-ethyl ester-containing alginate beads in HPMC capsules (size 0) is shown in Fig. 3. It was previously demonstrated that DTPA penta-ethyl ester exhibits $\mathrm{pH}$-dependent solubility and lipophilicity favorable for oral absorption, and that it is sufficiently stable at low $\mathrm{pH}$ to avoid extensive degradation in the gastrointestinal tract prior to absorption (Sueda et al., 2012). At pH 1.2, the HMPC capsules dissolved rapidly ( 4 $\min )$. After $20 \mathrm{~min}, 97 \pm 2 \%$ of the prodrug was solubilized in the dissolution medium. When neat DTPA pentaethyl ester was placed in the same type of HPMC capsule and dissolution medium at $37^{\circ} \mathrm{C}, 80 \pm 10 \%$ was solubilized after $20 \mathrm{~min}$ (data not shown). The dispersal of the prodrug in the matrix probably decreased hydrophobic interactions among the DTPA penta-ethyl ester molecules, in that drug was spread over a larger surface area, increasing the dissolution rate.

The following semi-empirical equation (Ritger \& Peppas, 1987;Siepmann \& Peppas, 2001) can be used to describe the release mechanism from swellable polymeric systems:

$$
\frac{M t}{M \infty}=k t^{n}
$$

In logarithmic form it is:

$$
\log \left(\frac{M t}{M \infty}\right)=\log k+n \log t
$$

where $M_{t} / M_{\infty}$ represents the fraction release of the drug at time $t, k$ is the release rate constant and $n$ is the release exponent indicative of the drug release mechanism. For spheres (Table 2), values of $n$ between 0.43 and 0.85 are an indication of anomalous transport. Values for $\mathrm{n}$ higher than 0.85 indicate case-II transport, which relates to polymer relaxation during hydrogel swelling, and values lower than 0.43 indicate that drug release is because of Fickian diffusion (Siepmann \& Peppas, 2001;Takka, Ocak, \& Acarturk, 1998). The values of the aforementioned terms in this system are listed in Table 2. The $n$ value is 0.529 , indicating that the release of DTPA penta-ethyl ester from the alginate beads is via anomalous transport. The anomalous transport is mainly controlled by the swelling of the alginate beads; free water penetrates the alginate beads via its pores and swells the matrix. Prodrug diffuses out through the beads with simultaneous polymer relaxation. The experimental data satisfy linear fitting up to 86\% DTPA penta-ethyl ester release, near the onset of the plateau, with a correlation coefficient $\left(I^{2}\right)$ of 0.997 .

\subsection{Stability and in vitro release in the presence of $0.6 \% \alpha$-tocopherol}

Because DTPA penta-ethyl ester is susceptible to oxidation and the hydrolysis of its ester functional groups, encapsulation of DTPA penta-ethyl ester is expected to enhance its stability by reducing its interactions with the environment. Neat DTPA penta-ethyl ester was placed in HPMC capsules with $0.6 \%$ a-tocopherol and stored at $4{ }^{\circ} \mathrm{C}, 25{ }^{\circ} \mathrm{C}$ and $40{ }^{\circ} \mathrm{C}$, all at approximately $80 \% \mathrm{RH}$. After three months, $77 \pm 2,68 \pm 1$ and $0.6 \pm 0.9 \%$ of DTPA penta-ethyl ester remained intact, respectively (Fig. 4A). DTPA penta-ethyl estercontaining 
alginate beads (formulation D3) with $0.6 \%$ a-tocopherol were placed in HPMC capsules and stored at the same temperatures and RH. After three months, $88 \pm 3,79 \pm 3$ and $51 \pm 2 \%$ of DTPA penta-ethyl ester remained intact, respectively (Fig. 4A). Thus, improved stability of the prodrug was observed after encapsulation in alginate beads.

\section{Conclusions}

The present study demonstrated that DTPA penta-ethyl ester, which exists as an oily liquid, can be made into a solid dosage form using alginate polymer and $\mathrm{CaCl}_{2}$ by ionotropic gelation. High content DTPA penta-ethyl ester-containing alginate beads were produced. The prodrug did not react with excipients during formation of DTPA penta-ethyl estercontaining alginate beads and increased stability of the prodrug was observed when encapsulated in the beads. Future efforts are being devoted to preparing a polymer-coated alginate bead to further protect the active ingredient from hydrolytic and oxidative degradation.

\section{Acknowledgments}

This work was funded by the National Institute of Health, U.S. Department of Health and Human Services under contracts HHSN266200500045C and HHSN272201000030C.

\section{References}

Bair WJ, Thompson RC. Plutonium: Biomedical research. Science. 1974; 183:715-722. [PubMed: 4595239]

Cassatt DR, Kaminski JM, Hatchett RJ, DiCarlo AL, Benjamin JM, Maidment BW. Medical countermeasures against nuclear threats: Radionuclide decorporation agents. Radiation Research. 2008; 170:540-548. [PubMed: 19024661]

Cekic ND, Savic SD, Milic J, Savic MM, Jovic Z, Malesevic M. Preparation and characterisation of phenytoin-loaded alginate and alginate-chitosan microparticles. Drug Delivery. 2007; 14:483-490. [PubMed: 18027177]

Chana ES. Preparation of Ca-alginate beads containing high oil content: Influence of process variables on encapsulation efficiency and bead properties. Carbohydrate Polymers. 2011; 84:1267-1275.

El-Kamel AH, Al-Gohary OM, Hosny EA. Alginate-diltiazem hydrochloride beads: Optimization of formulation factors, in vitro and in vivo availability. Journal of Microencapsulation. 2003; 20:211225. [PubMed: 12554376]

Han J, Guenier AS, Salmieri S, Lacroix M. Alginate and chitosan functionalization for micronutrient encapsulation. Journal of Agricultural and Food Chemistry. 2008; 56:2528-2535. [PubMed: 18324770]

Hari PR, Chandy T, Sharma CP. Chitosan/calcium alginate microcapsules for intestinal delivery of nitrofurantoin. Journal of Microencapsulation. 1996; 13:319-329. [PubMed: 8860687]

Luo Y, Zhang B, Whent M, Yu LL, Wang Q. Preparation and characterization of zein/chitosan complex for encapsulation of alpha-tocopherol, and its in vitro controlled release study. Colloids and Surfaces B: Biointerfaces. 2011; 85:145-152.

Manjanna KM, Pramod Kumar TM, Shivakumar B. Calcium alginate cross-linked polymeric microbeads for oral sustained drug delivery in arthritis. Drug Discoveries \& Therapeutics. 2010; 4:109-122. [PubMed: 22491168]

Østberg T, Graffner C. Calcium alginate matrices for oral multiple unit administration. III. Influence of calcium concentration, amount of drug added and alginate charateristics on drug release. International Journal of Pharmaceutics. 1994; 111:271-282.

Pongjanyakul T, Rongthong T. Enhanced entrapment efficiency and modulated drug release of alginate beads loaded with drug-clay intercalated complexes as microreservoirs. Carbohydrate Polymers. 2010; 81:409-419. 
Ramadas M, Paul W, Dileep KJ, Anitha Y, Sharma CP. Lipoinsulin encapsulated alginate-chitosan capsules: Intestinal delivery in diabetic rats. Journal of Microencapsulation. 2000; 17:405-411. [PubMed: 10898081]

Ritger PL, Peppas NA. A simple equation for description of solute release II. Fickian and anomalous release from swellable devices. Journal of Controlled Release. 1987; 5:37-42.

Sartori C, Finch DS, Ralph B. Determination of the cation content of alginate thin films by FTIR spectroscopy. Polymer. 1997; 38:43-51.

Siepmann J, Peppas NA. Modeling of drug release from delivery systems based on hydroxypropyl methylcellulose (HPMC). Advanced Drug Delivery Reviews. 2001; 48:139-157. [PubMed: 11369079]

Sueda K, Sadgrove MP, Fitzsimmons JM, Jay M. Physicochemical characterization of a prodrug of a radionuclide decorporation agent for oral delivery. Journal of Pharmaceutical Sciences. 2012; 101:2844-2853. [PubMed: 22644947]

Takka S, Ocak OH, Acarturk F. Formulation and investigation of nicardipine $\mathrm{HCl}$-alginate gel beads with factorial design-based studies. European Journal of Pharmaceutical Sciences. 1998; 6:241246. [PubMed: 9795073]

Wan LS, Heng PW, Chan LW. Drug encapsulation in alginate microspheres by emulsification. Journal of Microencapsulation. 1992; 9:309-316. [PubMed: 1403481]

Waterman KC, Adami RC, Alsante KM, Antipas AS, Arenson DR, Carrier R, et al. Hydrolysis in pharmaceutical formulations. Pharmaceutical Development and Technology. 2002; 7:113-146. [PubMed: 12066569] 

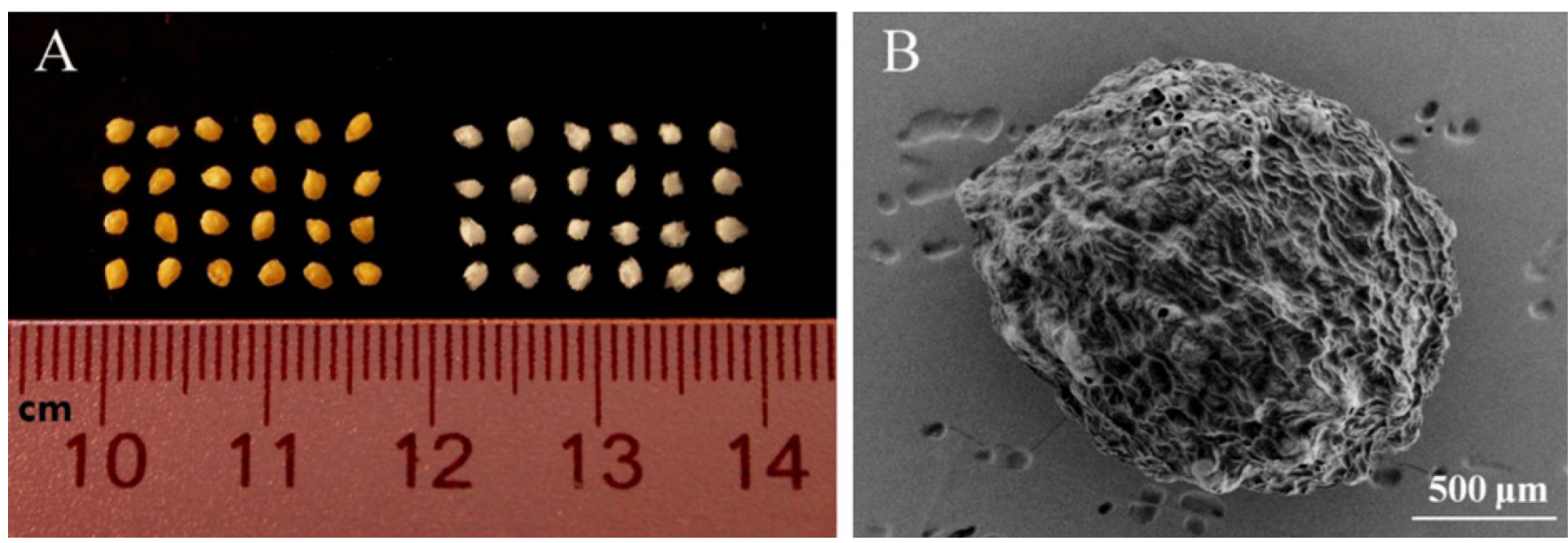

Fig. 1.

Photograph of DTPA penta-ethyl ester-containing alginate beads (left) and blank alginate beads (right) (A); SEM image of a DTPA penta-ethyl ester alginate bead (B). 


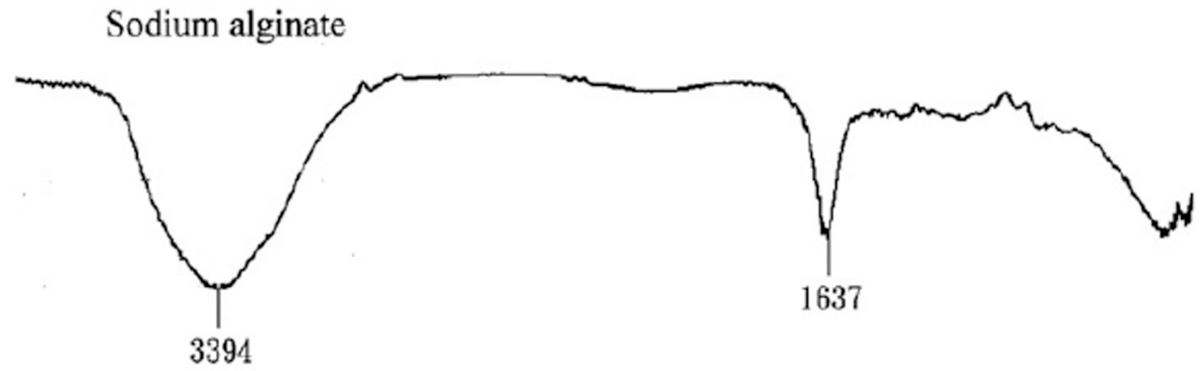

Alginate beads

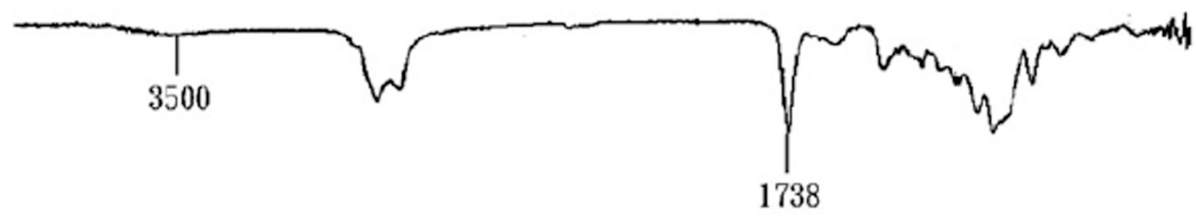

DTPA penta-ethyl ester-containing alginate beads

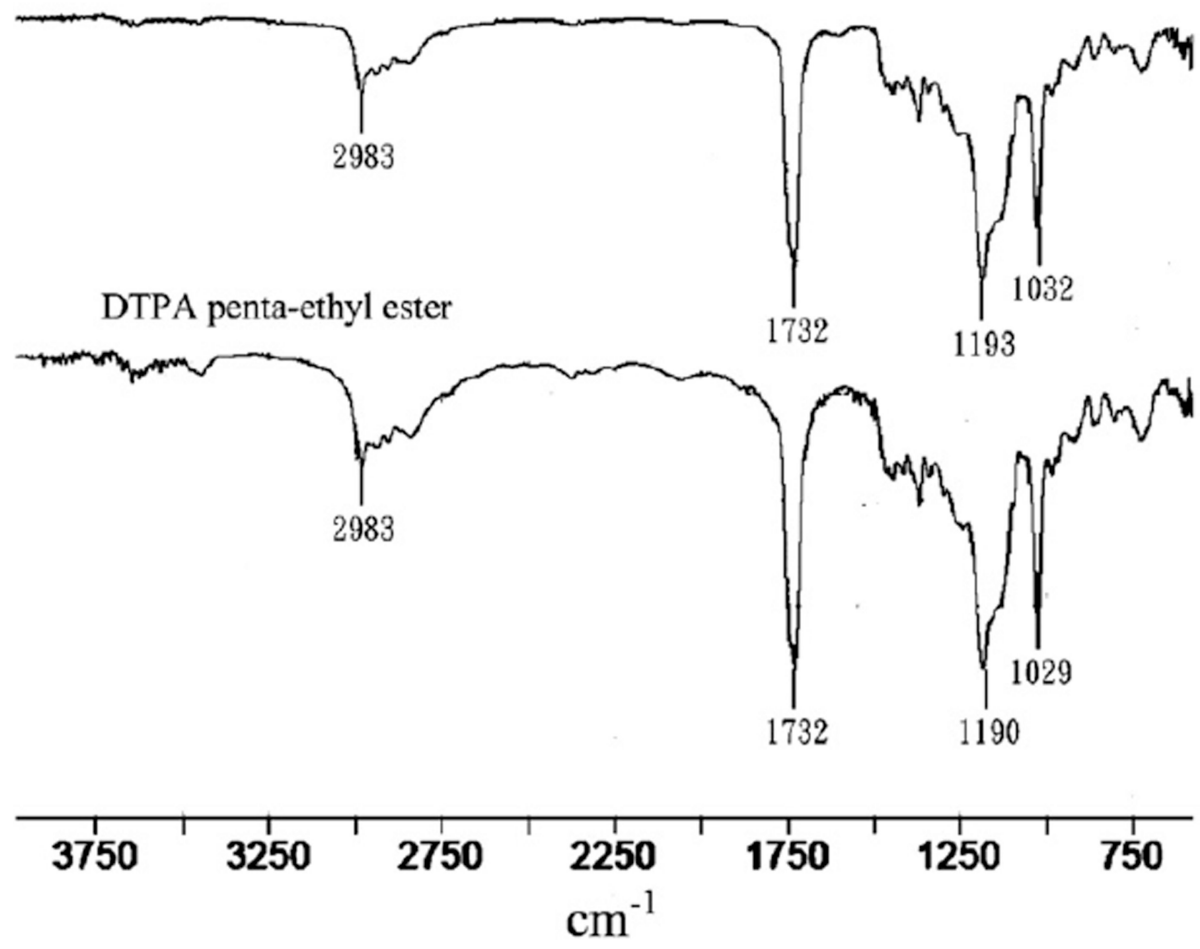

Fig. 2.

FTIR spectra of sodium alginate polymer, blank and DTPA penta-ethyl ester-containing alginate beads and neat DTPA penta-ethyl ester. 


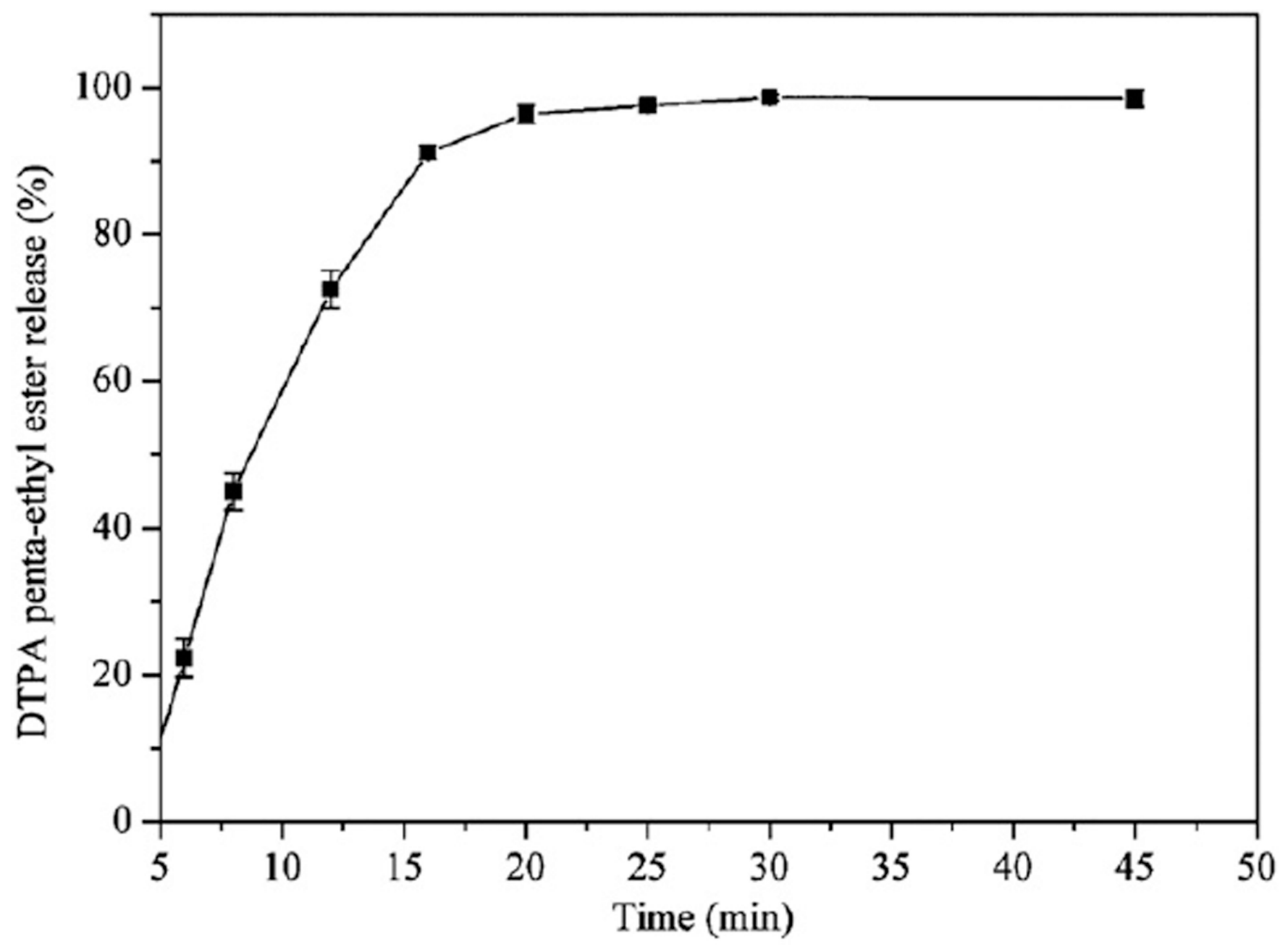

Fig. 3.

Percent of the DTPA penta-ethyl ester released and solubilized in dissolution medium $(0.1 \mathrm{~N}$ $\mathrm{HCl}, \mathrm{pH}$ 1.2) from alginate beads in HPMC capsules. Values are mean \pm standard deviation, $n=3$. 


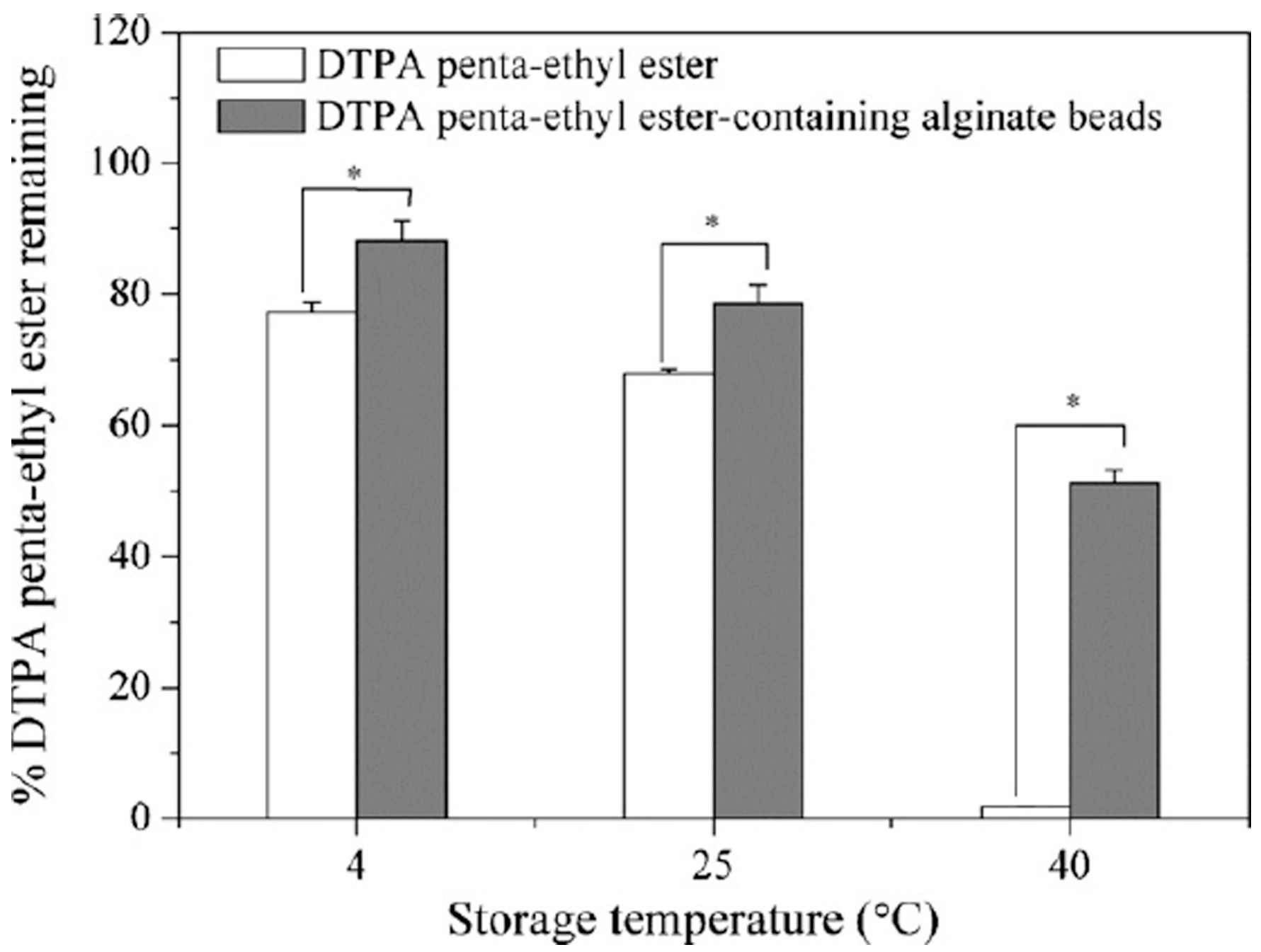

Fig. 4.

Stability of neat DTPA penta-ethyl ester with $0.6 \%$ a-tocopherol or DTPA penta-ethyl estercontaining alginate beads with $0.6 \%$ a-tocopherol in HPMC capsules after storage at $4{ }^{\circ} \mathrm{C}$, $25^{\circ} \mathrm{C}$ and $40{ }^{\circ} \mathrm{C}$ (all $\left.\sim 80 \% \mathrm{RH}\right)$ for three months. Values are mean \pm standard deviation, $n=$ 3. 
Table 2

Release mechanisms from polymeric delivery systems of different geometry and drug release mechanism of DTPA penta-ethyl ester from alginate beads.

\begin{tabular}{llll}
\hline Exponent, $\boldsymbol{n}$ & & & Drug release mechanism \\
Thin film & Cylinder & Sphere & \\
\hline 0.5 & 0.45 & 0.43 & Fickian diffusion \\
$0.5<n<1.0$ & $0.45<n<0.89$ & $0.43<n<0.85$ & Anomalous transport \\
1 & 0.89 & 0.85 & Case-II transport \\
Drug release mechanism of DTPA penta-ethyl ester from alginate beads ${ }^{a}$ \\
\hline$n$ & $k\left(\times 10^{2}\right)$ & $I^{2}$ & \\
\hline 0.529 & 28.89 & 0.997 & Anomalous transport \\
\hline
\end{tabular}

${ }^{a}$ Diffusional exponent $(n)$, kinetic constant $(k)$ and correlation coefficient $(r)$ by linear regression of $\log \left(M_{\mathrm{t}} / M_{\infty}\right)$ versus $\log t, k$ is the constant related to the structural and geometric characteristics of the device; $n$ is the diffusional exponent, which is indicative of release mechanism. 